\title{
The Schwartz Alternating Algorithm for Solving 3D Exterior Helmholtz Problems
}

\author{
Qing Chen, ${ }^{1}$ Baoqing Liu $\mathbb{D}^{2}{ }^{2}$ and Qikui Du' \\ ${ }^{1}$ School of Mathematical Sciences, Jiangsu Provincial Key Laboratory for NSLSCS, Nanjing Normal University, Nanjing 210023, China \\ ${ }^{2}$ School of Applied Mathematics, Nanjing University of Finance and Economics, Nanjing 210023, China
}

Correspondence should be addressed to Baoqing Liu; lyberal@163.com

Received 29 October 2017; Accepted 27 March 2018; Published 6 May 2018

Academic Editor: Jean Jacques Loiseau

Copyright (C) 2018 Qing Chen et al. This is an open access article distributed under the Creative Commons Attribution License, which permits unrestricted use, distribution, and reproduction in any medium, provided the original work is properly cited.

Based on the natural boundary reduction, an overlapping domain decomposition method is discussed for solving exterior Helmholtz problem over a three-dimensional (3D) domain. By introducing two different artificial boundaries, the original unbounded domain is divided into a bounded subdomain and a typical unbounded region, and a Schwartz alternating method is presented. The finite element method and natural boundary element method are alternately applied to solve the problems in the bounded subdomain and the typical unbounded subdomain. Moreover, the convergence of the Schwartz alternating algorithm is studied. Finally, some numerical experiments are presented to show the performance of this method.

\section{Introduction}

When solving a problem modelled by a linear partial differential equation in the bounded or unbounded domain, many existing methods can be adopted. For the problem in the bounded domain, the finite element method and the finite difference method and so on are very effective. Yet we often find them difficult to be applied to unbounded problem directly. To solve such problems in infinite region numerically, there are a variety of numerical methods (cf. [14]) and references therein for more details.

Schwartz alternating algorithm is one of the most efficient techniques for solving problems in the unbounded domain, such as harmonic equation [5, 6], Stokes equation [7], and Helmholtz equation [8]. Over exterior 3D domain, Wu and Yu studied the natural integral equations of Helmholtz problems [9] and overlapping domain decomposition method for harmonic equation [10]. We gave a D-N alternating algorithm for solving 3D exterior Helmholtz problems [11].

In this paper, a Schwartz alternating algorithm based on the natural boundary reduction is devised for the numerical solution of exterior three-dimensional Helmholtz problem. Firstly, an exterior Helmholtz problem is introduced and the corresponding variational form is obtained. Secondly, the Schwartz alternating algorithm is posed. Then, the convergence rate is discussed and the relationship between contraction factor $\alpha$ and overlapping extent is given. Besides, the error estimate of the algorithm is offered. Finally, some numerical examples are presented to illustrate the feasibility and efficiency of this method.

\section{Problem and Its Equivalent Form}

We consider the following 3D exterior Helmholtz problem [12]:

$$
\begin{aligned}
-\Delta u-k^{2} u & =0, \quad \text { in } \Omega^{c}, \\
u & =g, \quad \text { on } \Gamma_{0},
\end{aligned}
$$

where $\Omega$ is a bounded domain in $\mathbb{R}^{3}$ with regular boundary $\partial \Omega=\Gamma_{0} \cdot u(\mathbf{x})$ is the unknown function and $g$ is a known function, which satisfies appropriate conditions. $k$ denotes the wave number, related to the wavelength $\lambda$ of the incident wave through $k=2 \pi / \lambda$. In order to assure the existence and uniqueness of the solution of (1), the solution $u(\mathbf{x})$ satisfies the Sommerfeld radiation condition:

$$
\lim _{r \rightarrow \infty} r\left(\frac{\partial u}{\partial r}-\mathrm{i} k u\right)=0,
$$


where $(r, \theta, \varphi)$ denotes the spherical coordinates, $r=|\mathbf{x}|=$ $\sqrt{x^{2}+y^{2}+z^{2}}, \mathrm{i}=\sqrt{-1}$. Condition (2) asserts that the scattered wave is outgoing at infinity.

The corresponding variational form of problems (1)-(2) is

$$
\text { Find } u \in H^{1}\left(\Omega^{c}\right) \text {, }
$$

such that $a(u, v)=\langle g, v\rangle, \quad \forall v \in H_{0}^{1}\left(\Omega^{c}\right)$,

where

$$
\begin{aligned}
a(u, v) & =\int_{\Omega^{c}}\left(\nabla u \cdot \nabla v-k^{2} u v\right) \mathrm{d} \mathbf{x}, \\
\langle g, v\rangle & =\int_{\Gamma_{0}} g v \mathrm{~d} s, \\
H_{0}^{1}\left(\Omega^{c}\right) & =\left\{v \mid \frac{v}{\sqrt{1+r^{2}}}, \frac{\partial v}{\partial x}, \frac{\partial v}{\partial y}, \frac{\partial v}{\partial z} \in L^{2}\left(\Omega^{c}\right)\right\} .
\end{aligned}
$$

Particularly, if $\Omega$ is a spherical domain with radius $R$ whose centre is the origin of coordinates, the solution of (1)(2) is given by the following Poisson integral formula:

$$
\begin{gathered}
u(r, \theta, \varphi)=\mathscr{P} u(R, \theta, \varphi)=\sum_{\ell=0}^{\infty} \sum_{m=-\ell}^{\ell} \frac{h_{\ell}(k r)}{h_{\ell}(k R)} Y_{\ell}^{m}(\theta, \varphi) \\
\cdot \int_{0}^{2 \pi} \int_{0}^{\pi} u\left(R, \theta^{\prime}, \varphi^{\prime}\right) \overline{Y_{\ell}^{m}\left(\theta^{\prime}, \varphi^{\prime}\right)} \sin \theta^{\prime} \mathrm{d} \theta^{\prime} \mathrm{d} \varphi^{\prime},
\end{gathered}
$$

where $\mathscr{P}$ is called the Poisson integral operator of Helmholtz equation in $\Omega^{c}, h_{\ell}(x)=\sqrt{\pi / 2 x} H_{l+1 / 2}^{(1)}(x)$ is the first kind of Hankel function, $P_{\ell}^{m}(\cdot)$ denotes the associated Legendre function of the first kind, $\overline{Y_{\ell}^{m}(\cdot)}$ denotes the complex conjugate of $Y_{\ell}^{m}(\cdot)$, and

$$
\begin{aligned}
& Y_{\ell}^{m}(\theta, \varphi)=\sqrt{\frac{(\ell-m) !}{(\ell+m) !} \frac{2 \ell+1}{4 \pi}} P_{\ell}^{m}(\cos \theta) e^{i m \varphi}, \\
& P_{\ell}^{m}(x) \\
& \quad= \begin{cases}\frac{1}{2^{\ell} \ell !}\left(1-x^{2}\right)^{m / 2} \frac{d^{\ell+m}}{d x^{\ell+m}}\left(x^{2}-1\right)^{\ell}, & m \geqslant 0, \\
(-1)^{-m} \frac{(\ell+m) !}{(\ell-m) !} P_{\ell}^{-m}(x), & m<0 .\end{cases}
\end{aligned}
$$

By using (5), we will develop an overlapping domain decomposition method (Schwartz alternating algorithm based on the natural boundary reduction) for problems (1)-(2). Taking the normal derivative of (5), we have the following natural integral equation:

$$
\begin{array}{r}
\frac{\partial u}{\partial n}=\mathscr{K} u(R, \theta, \varphi)=-\sum_{\ell=0}^{\infty} \sum_{m=-\ell}^{\ell} \frac{k h_{\ell}^{\prime}(k R)}{h_{\ell}(k R)} Y_{\ell}^{m}(\theta, \varphi) \\
\cdot \int_{0}^{2 \pi} \int_{0}^{\pi} u\left(R, \theta^{\prime}, \varphi^{\prime}\right) \overline{Y_{\ell}^{m}\left(\theta^{\prime}, \varphi^{\prime}\right)} \sin \theta^{\prime} \mathrm{d} \theta^{\prime} \mathrm{d} \varphi^{\prime},
\end{array}
$$

where $\mathscr{K}$ is called the natural integral operator of Helmholtz equation in $\Omega^{c}$.

\section{Schwartz Alternating Algorithm and Its Convergence}

Taking two sphere surfaces $\Gamma_{1}=\left\{(r, \theta, \varphi) \mid r=R_{1}, 0 \leqslant \theta \leqslant\right.$ $\pi, 0 \leqslant \varphi \leqslant 2 \pi\}$ and $\Gamma_{2}=\left\{(r, \theta, \varphi) \mid r=R_{2}, 0 \leqslant \theta \leqslant \pi, 0 \leqslant\right.$ $\varphi \leqslant 2 \pi\}$, such that $R_{1}>R_{2}$ and $\Gamma_{0}$ is surrounded by $\Gamma_{2}$, denote

$$
\begin{aligned}
& \Omega_{1}=\left\{(x, y, z) \mid x^{2}+y^{2}+z^{2}<R_{1}^{2},(x, y, z) \in \Omega^{c}\right\}, \\
& \Omega_{2}=\left\{(x, y, z) \mid x^{2}+y^{2}+z^{2}>R_{2}^{2},(x, y, z) \in \Omega^{c}\right\}, \\
& \Omega_{11}=\Omega_{1} \cap \overline{\Omega_{2}^{c}}, \\
& \Omega_{12}=\Omega_{1} \cap \Omega_{2}, \\
& \Omega_{22}=\Omega_{2} \cap \overline{\Omega_{1}^{c}}, \\
& V=\left\{v \mid v \in H_{0}^{1}\left(\Omega^{c}\right), \lim _{r \rightarrow \infty} r\left(\frac{\partial v}{\partial r}-\mathrm{i} k v\right)=0\right\}, \\
& V_{1}=\left\{v\left|v \in H_{0}^{1}\left(\Omega_{1}\right), v\right|_{\Gamma_{1}}=0\right\}, \\
& V_{2}=\left\{v\left|v \in H_{0}^{1}\left(\Omega_{2}\right), v\right|_{\Gamma_{2}}=0, \lim _{r \rightarrow \infty} r\left(\frac{\partial v}{\partial r}-\mathrm{i} k v\right)\right. \\
& \quad=0\}, \\
& V_{n}=\left\{v\left|v \in H_{0}^{1}\left(\Omega_{1}\right), v\right|_{\Gamma_{1}}=\lambda^{(n)}\right\} .
\end{aligned}
$$

Construct the following Schwartz alternating algorithm. Step 1. Select the initial $u_{0} \in V$ and let $\eta^{(0)}=\left.u_{0}\right|_{\Gamma_{1}}, n=0$.

Step 2. Solve the problem in $\Omega_{1}$ :

$$
\begin{aligned}
-\Delta u_{1}^{(2 n+1)}-k^{2} u_{1}^{(2 n+1)} & =0, \quad \text { in } \Omega_{1}, \\
u_{1}^{(2 n+1)} & =\eta^{(n)}, \quad \text { on } \Gamma_{1}, \\
u_{1}^{(2 n+1)} & =g, \quad \text { on } \Gamma_{0} .
\end{aligned}
$$

Step 3. Solve the problem in $\Omega_{2}$ :

$$
\begin{aligned}
-\Delta u_{2}^{(2 n+2)}-k^{2} u_{2}^{(2 n+2)} & =0, \quad \text { in } \Omega_{2}, \\
u_{2}^{(2 n+2)} & =u_{1}^{(2 n+1)}, \quad \text { on } \Gamma_{2}, \\
\lim _{r \rightarrow \infty} r\left(\frac{\partial u_{2}^{(2 n+2)}}{\partial r}-i k u_{2}^{(2 n+2)}\right) & =0 .
\end{aligned}
$$

Step 4. Let $\eta^{(n+1)}=\left.u_{2}^{(2 n+2)}\right|_{\Gamma_{1}}$.

Step 5. Let $n=n+1$, and go to Step 2 .

Where $u_{1}^{(2 n+1)}$ and $u_{2}^{(2 n+2)}$ are the $2 n+1$ th and $2 n+2$ th approximate solutions in $\Omega_{1}$ and $\Omega_{2}$, respectively, $\eta^{(0)}$ is an arbitrary function in $H^{1 / 2}\left(\Gamma_{1}\right)$. Note that, on interface $\Gamma_{1}$, only $\eta^{(n+1)}=\left.u_{2}^{(2 n+2)}\right|_{\Gamma_{1}}$ is needed. So it is unnecessary to solve (10). 
Actually we can obtain $\eta^{(n+1)}$ by making use of the following Poisson integral equation:

$$
\eta^{(n+1)}=\left.u_{2}^{(2 n+2)}\right|_{\Gamma_{1}}=\left.\mathscr{P}\left(\gamma_{2} u_{1}^{(2 n+1)}\right)\right|_{\Gamma_{1}},
$$

where $\gamma_{2}$ is the trace operator on $\Gamma_{2}$. Because of $u_{2}^{(2 n)} \in V_{2}$ and $u_{1}^{(2 n+1)} \in V_{1}, V_{1}$ and $V_{2}$ can be regarded as the subspaces of $V$, if elements of $V$ are extended by zero. Let $\left.u_{1}^{(2 n+1)}\right|_{\Omega_{22}}=u_{2}^{(2 n)}$ and $\left.u_{2}^{(2 n)}\right|_{\Omega_{11}}=u_{1}^{(2 n-1)}$; obviously $u^{(\ell)} \in V, \ell=0,1,2, \ldots$, $u_{1}^{(2 n+1)}-u_{2}^{(2 n)} \in V_{1}$, and $u_{2}^{(2 n+2)}-u_{1}^{(2 n+1)} \in V_{2}$. (9) and (10) are, respectively, equivalent to the following variational forms:

Find $u_{1}^{(2 n+1)}-u_{2}^{(2 n)} \in V_{1}$,

such that $a\left(u_{1}^{(2 n+1)}-u, v_{1}\right)=0, \quad \forall v_{1} \in V_{1}$.

Find $u_{2}^{(2 n)}-u_{1}^{(2 n-1)} \in V_{2}$,

such that $a\left(u_{2}^{(2 n)}-u, v_{2}\right)=0, \quad \forall v_{2} \in V_{2}$.

$P_{V_{i}}$ denotes $V$ projected to $V_{i}$; change (12) into

$$
\begin{aligned}
a\left(u_{1}^{(2 n+1)}-u_{2}^{(2 n)}, v_{1}\right)= & a\left(u-u_{2}^{(2 n)}, v_{1}\right), \\
& \forall v_{1} \in V_{1}, u_{1}^{(2 n+1)}-u_{2}^{(2 n)} \in V_{1}, \\
a\left(u_{2}^{(2 n)}-u_{1}^{(2 n-1)}, v_{2}\right)= & a\left(u-u_{1}^{(2 n-1)}, v_{2}\right), \\
& \forall v_{2} \in V_{2}, u_{2}^{(2 n)}-u_{1}^{(2 n-1)} \in V_{2} .
\end{aligned}
$$

By the definition of projection, we obtain

$$
\begin{aligned}
& u_{1}^{(2 n+1)}-u_{2}^{(2 n)}=P_{V_{1}}\left(u-u_{2}^{(2 n)}\right), \quad n \geqslant 0, \\
& u_{2}^{(2 n)}-u_{1}^{(2 n-1)}=P_{V_{2}}\left(u-u_{1}^{(2 n-1)}\right), \quad n \geqslant 1 .
\end{aligned}
$$

This is equivalent to

$$
\begin{gathered}
u-u_{1}^{(2 n+1)}=P_{V_{1}^{\perp}}\left(u-u_{2}^{(2 n)}\right), \quad n \geqslant 0, \\
u-u_{2}^{(2 n)}=P_{V_{2}^{\perp}}\left(u-u_{1}^{(2 n-1)}\right), \quad n \geqslant 1,
\end{gathered}
$$

where $V_{i}^{\perp}$ is the orthogonal complementary space of $V_{i}$; that is,

$$
P_{V_{i}^{\perp}}=I-P_{V_{i}}, \quad i=1,2 .
$$

Let $e_{i}^{(j)}=u-u_{i}^{(j)}(i=1,2)$ denote the errors. Then (15) can be rewritten as

$$
\begin{aligned}
e_{1}^{(2 n+1)} & =P_{V_{1}^{\perp}} e_{2}^{(2 n)}, \\
e_{2}^{(2 n)} & =P_{V_{2}^{\perp}} e_{1}^{(2 n-1)} .
\end{aligned}
$$

This yields

$$
\begin{aligned}
& e_{1}^{(2 n+1)}=P_{V_{2}^{\perp}} P_{V_{1}^{\perp}} e_{1}^{(2 n-1)}, \\
& e_{2}^{(2 n+2)}=P_{V_{1}^{\perp}} P_{V_{2}} e_{2}^{(2 n)} .
\end{aligned}
$$

It is easy to know that if $e_{i}^{(j)}(i=1,2)$ are convergent, then their limits are in $V_{1}^{\perp} \cap V_{2}^{\perp}$.
Lemma 1. $V=V_{1}+V_{2}, V_{1}^{\perp} \cap V_{2}^{\perp}=\{0\}$.

Lemma 2. $V=V_{1}+V_{2}$ and for any $v \in V$ there exists a positive constant $C_{0}$ such that

$$
|v|_{1} \leqslant C_{0}\left(\left|P_{V_{1}}\right|_{1}^{2}+\left|P_{V_{2}}\right|_{1}^{2}\right)^{1 / 2}
$$

where $|\cdot|_{1}=\sqrt{a(\cdot, \cdot)}$ denotes energy norm.

Proof. If $V=V_{1}+V_{2}$, there exists a positive constant $C_{0}$ such that

$$
\begin{aligned}
\left(\left|v_{1}\right|_{1}^{2}+\left|v_{2}\right|_{1}^{2}\right)^{1 / 2} & \leqslant C_{0}|v|_{1}, \\
v & =v_{1}+v_{2},
\end{aligned}
$$

$$
v_{1} \in V_{1}, \quad v_{2} \in V_{2} .
$$

Simultaneously,

$$
\begin{aligned}
|v|_{1}^{2} & =a\left(v, v_{1}\right)+a\left(v, v_{2}\right)=a\left(P_{V_{1}} v, v_{1}\right)+a\left(P_{V_{2}} v, v_{2}\right) \\
& \leqslant\left(\left|P_{V_{1}} v\right|_{1}^{2}+\left|P_{V_{2}} v\right|_{1}^{2}\right)^{1 / 2}\left(\left|v_{1}\right|_{1}^{2}+\left|v_{2}\right|_{1}^{2}\right)^{1 / 2} \\
& \leqslant C_{0}|v|_{1}\left(\left|P_{V_{1}} v\right|_{1}^{2}+\left|P_{V_{2}} v\right|_{1}^{2}\right)^{1 / 2} .
\end{aligned}
$$

Theorem 3. Suppose that $V_{1}^{\perp} \cap V_{2}^{\perp}=\{0\}$; then

$$
\begin{gathered}
\lim _{n \rightarrow+\infty}\left|e_{1}^{(2 n+1)}\right|_{1}=0, \\
\lim _{n \rightarrow+\infty}\left|e_{2}^{(2 n)}\right|_{1}=0 .
\end{gathered}
$$

Proof. Following (15), we have

$$
\left|e_{1}^{(2 n+1)}\right|_{1} \geqslant\left|e_{2}^{(2 n)}\right|_{1} \geqslant\left|e_{1}^{(2 n+3)}\right|_{1} \geqslant\left|e_{2}^{(2 n+2)}\right|_{1} \geqslant 0,
$$

which means that

$$
\lim _{n \rightarrow+\infty}\left|e_{1}^{(2 n+1)}-e_{2}^{(2 n)}\right|_{1}=0 .
$$

Let $\left\{e_{1}^{(2 n+1)}\right\}$ and $\left\{e_{2}^{(2 n)}\right\}$ be weakly convergent subsequence, so

$$
\begin{gathered}
\lim _{n \rightarrow+\infty} e_{1}^{(2 n+1)}=v_{1}, \\
\lim _{n \rightarrow+\infty} e_{2}^{(2 n)}=v_{2} .
\end{gathered}
$$

Following (24), we can obtain

$$
v_{1}=v_{2}=0 \text {. }
$$

Following (17), we have

$$
\begin{aligned}
a\left(e_{1}^{(2 n+1)}, e_{1}^{(2 n+1)}\right) & =a\left(e_{1}^{(2 n-1)}, e_{1}^{(2 n+3)}\right)=\cdots \\
& =a\left(e_{1}^{(4 n+1)}, e_{1}^{(1)}\right) \longrightarrow 0, \\
a\left(e_{2}^{(2 n)}, e_{2}^{(2 n)}\right) & =a\left(e_{2}^{(2 n-2)}, e_{2}^{(2 n+2)}\right)=\cdots \\
& =a\left(e_{2}^{(4 n)}, e_{2}^{(0)}\right) \longrightarrow 0 ;
\end{aligned}
$$


namely

$$
\begin{gathered}
\lim _{n \rightarrow+\infty}\left|e_{1}^{(2 n+1)}\right|_{1}=0 \\
\lim _{n \rightarrow+\infty}\left|e_{2}^{(2 n)}\right|_{1}=0
\end{gathered}
$$

Theorem 4. There exists a constant $\alpha \in[0,1)$, such that

$$
\begin{aligned}
& \left|P_{V_{1}^{\perp}} P_{V_{2}^{\perp}}\right| \leqslant \alpha, \\
& \left|P_{V_{2}^{\perp}} P_{V_{1}^{\perp}}\right| \leqslant \alpha, \\
& \left|e_{1}^{(2 n+1)}\right|_{1} \leqslant \alpha^{2 n}\left|e_{1}^{(1)}\right|_{1}, \\
& \left|e_{2}^{(2 n+2)}\right|_{1} \leqslant \alpha^{2 n+2}\left|e_{2}^{(0)}\right|_{1}
\end{aligned}
$$

hold true.

Proof. Substituting $P_{V_{1}^{\perp}} v$ into (19), we have

$$
\begin{aligned}
\left|P_{V_{1}^{\perp}} v\right|_{1}^{2} & =\left|P_{V_{2}^{\perp}} P_{V_{1}^{\perp}} v\right|_{1}^{2}+\left|P_{V_{2}} P_{V_{1}^{\perp}} v\right|_{1}^{2} \\
& \geqslant\left|P_{V_{2}^{\perp}} P_{V_{1}^{\perp}} v\right|_{1}^{2}+\frac{1}{C_{0}^{2}}\left|P_{V_{1}^{\perp}} v\right|_{1}^{2} .
\end{aligned}
$$

Equivalently

$$
\left|P_{V_{2}^{\perp}} P_{V_{1}^{\perp}} v\right|_{1}^{2} \leqslant\left(1-\frac{1}{C_{0}^{2}}\right)\left|P_{V_{1}^{\perp}} v\right|_{1}^{2} \leqslant \alpha|v|_{1}^{2}, \quad \forall v \in V,
$$

where

$$
\alpha=\sqrt{1-\frac{1}{C_{0}^{2}}} \in[0,1)
$$

namely

$$
\left|P_{V_{2}^{\perp}} P_{V_{1}^{\perp}}\right| \leqslant \alpha
$$

Similarly

$$
\left|P_{V_{1}^{\perp}} P_{V_{2}^{\perp}}\right| \leqslant \alpha
$$

Following (17) and (18), it comes that

$$
\begin{aligned}
\left|e_{1}^{(2 n+1)}\right| & \leqslant \alpha\left|P_{V_{1}^{\perp}} e^{(2 n-1)}\right|_{1}=\alpha\left|P_{V_{1}^{\perp}} P_{V_{2}^{\perp}} e^{(2 n-2)}\right|_{1} \\
& \leqslant \alpha^{2}\left|P_{V_{2}^{\perp}} e^{(2 n-2)}\right|_{1}=\alpha^{2}\left|e^{(2 n-1)}\right|_{1} .
\end{aligned}
$$

By recursion we obtain

$$
\left|e_{1}^{(2 n+1)}\right|_{1} \leqslant \alpha^{2 n}\left|e_{1}^{(1)}\right|_{1} .
$$

Similarly, we obtain

$$
\left|e_{2}^{(2 n+2)}\right|_{1} \leqslant \alpha^{2 n+2}\left|e_{2}^{(0)}\right|_{1} .
$$

\section{Analysis of Convergence Rate}

Absolutely, the convergence rate of the above Schwartz alternating algorithm is closely related to the overlapping degree of $\Omega_{1}$ and $\Omega_{2}$. Although it can be deduced intuitively that the larger the overlapping part is, the faster the convergence rate will be; yet we find it difficult to analyse the convergence rate for general unbounded domain $\Omega^{c}$. However, under certain assumptions, we can find out the relationship between contraction factor $\alpha$ and overlapping degree of $\Omega_{1}$ and $\Omega_{2}$.

Consider the following boundary value problem in domain $\Omega_{1}$ :

$$
\begin{aligned}
& -\Delta u-k^{2} u=0, \quad \text { in } \Omega_{1} \text {, } \\
& u=f, \quad \text { on } \Gamma_{1} \text {, } \\
& u=g \text {, on } \Gamma_{0} \text {. }
\end{aligned}
$$

Lemma 5. If $f \in H^{1 / 2}\left(\Gamma_{1}\right)$ and $g \in H^{1 / 2}\left(\Gamma_{0}\right)$, then $u$ is the solution of (38).

$$
\begin{aligned}
u= & \sum_{\ell=0}^{\infty} \sum_{m=-\ell}^{\ell} \frac{1}{\left(R_{1}^{2 \ell+1}-R^{2 \ell+1}\right) r^{\ell+1}} \\
& \cdot\left[\left(R_{1}^{2 \ell+1}-r^{2 \ell+1}\right) R^{\ell+1} \frac{h_{\ell}(k r)}{h_{\ell}(k R)} G_{\ell}^{m}\right. \\
& \left.+\left(r^{2 \ell+1}-R^{2 \ell+1}\right) R_{1}^{\ell+1} \frac{h_{\ell}(k r)}{h_{\ell}\left(k R_{1}\right)} F_{\ell}^{m}\right] Y_{\ell}^{m}(\theta, \varphi),
\end{aligned}
$$

where

$$
\begin{aligned}
& F_{\ell}^{m}=\int_{0}^{2 \pi} \int_{0}^{\pi} f\left(R_{1}, \theta, \varphi\right) \overline{Y_{\ell}^{m}(\theta, \varphi)} \sin \theta \mathrm{d} \theta \mathrm{d} \varphi, \\
& G_{\ell}^{m}=\int_{0}^{2 \pi} \int_{0}^{\pi} g(R, \theta, \varphi) \overline{Y_{\ell}^{m}(\theta, \varphi)} \sin \theta \mathrm{d} \theta \mathrm{d} \varphi .
\end{aligned}
$$

Theorem 6. If we apply the Schwartz alternating algorithm given in Section 3 to problems (1)-(2) and $u_{0} \in H^{1 / 2}\left(\Gamma_{0}\right)$, then

$$
\begin{gathered}
\left\|u-u_{1}^{(2 n)}\right\|_{H^{1}\left(\Omega_{1}\right)} \leqslant C_{1} \alpha^{n}, \\
\left\|u-u_{2}^{(2 n+1)}\right\|_{W_{0}^{1}\left(\Omega_{2}\right)} \leqslant C_{2} \alpha^{n+1}
\end{gathered}
$$

hold true, where constants $C_{1}$ and $C_{2}$ are independent of $n$, while

$$
\alpha=\frac{R_{2}-R}{R_{1}-R}
$$

Proof. On one hand, for $u_{0} \in H^{1 / 2}\left(\Gamma_{0}\right)$, we have

$$
\begin{gathered}
u_{0}(R, \theta, \varphi)=\sum_{\ell=0}^{\infty} \sum_{m=-\ell}^{\ell} \frac{h_{\ell}(k R)}{h_{\ell}(k R)} U_{\ell}^{m} Y_{\ell}^{m}(\theta, \varphi) \\
=\sum_{\ell=0}^{\infty} \sum_{m=-\ell}^{\ell} U_{\ell}^{m} Y_{\ell}^{m}(\theta, \varphi), \\
\sum_{\ell=0}^{\infty} \sum_{m=-\ell}^{\ell}\left(1+\ell^{2}\right)^{1 / 2}\left|U_{\ell}^{m}\right|^{2}<+\infty,
\end{gathered}
$$


where

$$
U_{\ell}^{m}=\int_{0}^{2 \pi} \int_{0}^{\pi} u_{0}(R, \theta, \varphi) \overline{Y_{\ell}^{m}(\theta, \varphi)} \sin \theta \mathrm{d} \theta \mathrm{d} \varphi .
$$

Following [9], we obtain

$$
u(r, \theta, \varphi)=\sum_{\ell=0}^{\infty} \sum_{m=-\ell}^{\ell} \frac{h_{\ell}(k r)}{h_{\ell}(k R)} U_{\ell}^{m} Y_{\ell}^{m}(\theta, \varphi), \quad r>R .
$$

On the other hand, for $\tilde{u} \in W_{0}^{1}\left(\Omega^{c}\right)$, we have $\left.\widetilde{u}\right|_{\Gamma_{1}} \in H^{1 / 2}\left(\Gamma_{1}\right)$, while

$$
\begin{aligned}
\left.\widetilde{u}\right|_{\Gamma_{1}} & =\sum_{\ell=0}^{\infty} \sum_{m=-\ell}^{\ell} \widetilde{U}_{\ell}^{m} Y_{\ell}^{m}(\theta, \varphi), \\
\sum_{\ell=0}^{\infty} \sum_{m=-\ell}^{\ell}\left(1+\ell^{2}\right)^{-1 / 2}\left|\widetilde{U}_{\ell}^{m}\right|^{2}<+\infty, &
\end{aligned}
$$

where

$$
\widetilde{U}_{\ell}^{m}=\int_{0}^{2 \pi} \int_{0}^{\pi} \widetilde{u}\left(R_{1}, \theta, \varphi\right) \overline{Y_{\ell}^{m}(\theta, \varphi)} \sin \theta \mathrm{d} \theta \mathrm{d} \varphi .
$$

According to Lemma 5, it comes that

$$
\begin{gathered}
u_{1}^{(2 n)}=\sum_{\ell=0}^{\infty} \sum_{m=-\ell}^{\ell}\left(\frac{R^{\ell+1} U_{\ell}^{m}}{r^{\ell+1}}-\alpha_{\ell}^{n} \frac{r^{2 \ell+1}-R^{2 \ell+1}}{R_{1}^{2 \ell+1}-R^{2 \ell+1}}\right. \\
\left.\cdot \frac{R^{\ell+1} U_{\ell}^{m}-R_{1}^{\ell+1} \widetilde{U}_{\ell}^{m}}{r^{\ell+1}}\right) \frac{h_{\ell}(k r)}{h_{\ell}(k R)} Y_{\ell}^{m}(\theta, \varphi),
\end{gathered}
$$

where

$$
\alpha_{\ell}=\frac{R_{2}^{2 \ell+1}-R^{2 \ell+1}}{R_{1}^{2 \ell+1}-R^{2 \ell+1}} .
$$

Suppose that $n \in \overline{Z^{-}}$; we have

$$
\begin{gathered}
\left.u_{1}^{(2 n)}\right|_{\Gamma_{2}}=\sum_{\ell=0}^{\infty} \sum_{m=-\ell}^{\ell}\left(\frac{R^{\ell+1} U_{\ell}^{m}}{R_{2}{ }^{\ell+1}}\right. \\
\left.-\alpha_{\ell}^{n+1} \frac{R^{\ell+1} U_{\ell}^{m}-R_{1}^{\ell+1} \widetilde{U}_{\ell}^{m}}{R_{2}^{\ell+1}}\right) \frac{h_{\ell}\left(k R_{2}\right)}{h_{\ell}(k R)} Y_{\ell}^{m}(\theta, \varphi), \\
u_{2}^{(2 n+1)}=\sum_{\ell=0}^{\infty} \sum_{m=-\ell}^{\ell}\left(\frac{R^{\ell+1} U_{\ell}^{m}}{R_{2}^{\ell+1}}-\alpha_{\ell}^{n+1}\right. \\
\left.\cdot \frac{R^{\ell+1} U_{\ell}^{m}-R_{1}^{\ell+1} \widetilde{U}_{\ell}^{m}}{R_{2}^{\ell+1}}\right) \frac{R_{2}^{l+1}}{r^{l+1}} \frac{h_{\ell}(k r)}{h_{\ell}(k R)} Y_{\ell}^{m}(\theta, \varphi) .
\end{gathered}
$$

Following trace theorem, we have

$$
\begin{aligned}
\left.u_{2}^{(2 n+1)}\right|_{\Gamma_{1}} & \\
= & \sum_{\ell=0}^{\infty} \sum_{m=-\ell}^{\ell}\left(\frac{R^{\ell+1} U_{\ell}^{m}}{R_{1}^{\ell+1}}-\alpha_{\ell}^{n+1} \frac{R^{\ell+1} U_{\ell}^{m}-R_{1}^{\ell+1} \widetilde{U}_{\ell}^{m}}{R_{1}^{\ell+1}}\right) \\
& \cdot \frac{h_{\ell}\left(k R_{1}\right)}{h_{\ell}(k R)} Y_{\ell}^{m}(\theta, \varphi) .
\end{aligned}
$$

By Lemma 5 again, we obtain

$$
\begin{gathered}
u_{1}^{(2 n+2)}=\sum_{\ell=0}^{\infty} \sum_{m=-\ell}^{\ell}\left(\frac{R^{\ell+1} U_{\ell}^{m}}{r^{\ell+1}}-\alpha_{\ell}^{n+1} \frac{r^{2 \ell+1}-R^{2 \ell+1}}{R_{1}^{2 \ell+1}-R^{2 \ell+1}}\right. \\
\left.\frac{R^{\ell+1} U_{\ell}^{m}-R_{1}^{\ell+1} \widetilde{U}_{\ell}^{m}}{r^{\ell+1}}\right) \frac{R_{2}^{\ell+1}}{r^{\ell+1}} \frac{h_{\ell}(k r)}{h_{\ell}(k R)} Y_{\ell}^{m}(\theta, \varphi) .
\end{gathered}
$$

By induction, it comes that

$$
\begin{aligned}
&\left.u\right|_{\Omega_{1}}-u_{1}^{(2 n)}= \sum_{\ell=0}^{\infty} \sum_{m=-\ell}^{\ell} \alpha_{\ell}^{n} \frac{r^{2 \ell+1}-R^{2 \ell+1}}{R_{1}^{2 \ell+1}-R^{2 \ell+1}} \\
& \cdot \frac{R^{\ell+1} U_{\ell}^{m}-R_{1}^{\ell+1} \widetilde{U}_{\ell}^{m}}{r^{\ell+1}} \frac{R_{2}^{\ell+1}}{r^{\ell+1}} \frac{h_{\ell}(k r)}{h_{\ell}\left(k R_{1}\right)} Y_{\ell}^{m}(\theta, \varphi) .
\end{aligned}
$$

Denoting

$$
\begin{aligned}
e_{N}^{(2 n)} & =\sum_{\ell=0}^{N} \sum_{m=-\ell}^{\ell} \alpha_{\ell}^{n} \\
& \cdot \frac{r^{2 \ell+1}-R^{2 \ell+1}}{R_{1}^{2 \ell+1}-R^{2 \ell+1}} \frac{R^{\ell+1} U_{\ell}^{m}-R_{1}^{\ell+1} \widetilde{U}_{\ell}^{m}}{r^{\ell+1}} \frac{R_{2}^{\ell+1}}{r^{\ell+1}} \frac{h_{\ell}(k r)}{h_{\ell}\left(k R_{1}\right)} Y_{\ell}^{m}(\theta,
\end{aligned}
$$

$\varphi)$,

(53) is changed into

$$
\lim _{N \rightarrow+\infty}\left|e_{N}^{(2 n)}-\left(u-u_{1}^{(2 n)}\right)\right|_{H^{1}\left(\Omega_{1}\right)}=0 .
$$

Clearly

$$
\Delta e_{N}^{(2 n)}=0
$$

noting that

$$
0<\alpha_{\ell}=\frac{R_{2}^{2 \ell+1}-R^{2 \ell+1}}{R_{1}^{2 \ell+1}-R^{2 \ell+1}} \leqslant \frac{R_{2}-R}{R_{1}-R}=\alpha, \quad \ell=0,1, \ldots
$$

Following (56) and (57), there exists $C_{1}$ independent of $n$, such that

$$
\left\|e_{N}^{(2 n)}\right\|_{H^{1}\left(\Omega_{1}\right)} \leqslant C_{1} \alpha^{n}
$$

Similarly, we obtain

$$
\left\|e_{N}^{(2 n)}\right\|_{W_{0}^{1}\left(\Omega_{2}\right)} \leqslant C_{2} \alpha^{n+1}
$$

It can be seen from Theorem 6 that the larger the overlapping part of $\Omega_{1}$ and $\Omega_{2}$ is, the smaller the contraction factor $\alpha$ is and, consequently, the faster the Schwartz alternating algorithm converges.

\section{Error Estimates of Algorithms}

Subdivide $\Omega_{1}$ into hexahedrons. Let $S_{h}\left(\Omega_{1}\right)$ denote the linear finite element space over $\Omega_{1}$. Putting

$$
\stackrel{\circ}{S}_{h}\left(\Omega_{1}\right)=\left\{v_{h}\left|v_{h} \in S_{h}\left(\Omega_{1}\right), v_{h}\right|_{\Gamma_{0} \cup \Gamma_{1}}=0\right\},
$$


$\stackrel{\circ}{S}_{h}\left(\Omega_{1}\right)$ can be regarded as the subspace of $V$ if its elements are extended by zero. We first establish the following discrete Schwartz alternating algorithm:

Find $\quad w_{1 h}^{(2 n+1)}=u_{1 h}^{(2 n+1)}-u_{h}^{(2 n)} \in \stackrel{\circ}{S}_{h}\left(\Omega_{1}\right)$,

such that $a\left(w_{1 h}^{(2 n+1)}, v_{h}\right)=-a\left(u_{h}^{(2 n)}, v_{h}\right)$,

$$
\forall v_{h} \in \stackrel{\circ}{S}_{h}\left(\Omega_{1}\right),
$$

Find $\quad w_{2 h}^{(2 n+2)}=u_{2 h}^{(2 n+2)}-u_{h}^{(2 n+1)} \in V_{2}$,

such that $a\left(w_{2 h}^{(2 n+2)}, v\right)=-a\left(u_{h}^{(2 n+1)}, v\right)$,

$\forall v_{h} \in V_{2}$,

with

$$
\begin{gathered}
u_{h}^{(2 n+1)}=\tilde{u}+ \begin{cases}\sum_{i=0}^{n} w_{1 h}^{(2 i+1)}, & \text { on } \bar{\Omega} \backslash \Omega_{2}, \\
\sum_{i=0}^{n} w_{1 h}^{(2 i+1)}+\sum_{j=0}^{n-1}\left[\mathscr{P} \gamma w_{1 h}^{(2 j+1)}-w_{1 h}^{(2 j+1)}\right]+\varepsilon_{n}(\mathscr{P} \gamma \tilde{u}-\tilde{u}), & \text { in } \Omega_{1} \backslash\left(\bar{\Omega} \backslash \Omega_{2}\right), \\
\sum_{j=0}^{n-1} \mathscr{P} \gamma w_{1 h}^{(2 j+1)}+\varepsilon_{n}(\mathscr{P} \gamma \tilde{u}-\tilde{u}), & \text { on } \Omega \backslash \Omega_{1},\end{cases} \\
u_{h}^{(2 n+2)}=\tilde{u}+ \begin{cases}\sum_{i=0}^{n} w_{1 h}^{(2 i+1)}, & \text { on } \bar{\Omega} \backslash \Omega_{2}, \\
\sum_{i=0}^{n} w_{1 h}^{(2 i+1)}+\sum_{j=0}^{n-1}\left[\mathscr{P} \gamma w_{1 h}^{(2 j+1)}-w_{1 h}^{(2 j+1)}\right]+(\mathscr{P} \gamma \widetilde{u}-\widetilde{u}), & \text { in } \Omega_{1} \backslash\left(\bar{\Omega} \backslash \Omega_{2}\right), \\
\sum_{j=0}^{n-1} \mathscr{P} \gamma w_{1 h}^{(2 j+1)}+(\mathscr{P} \gamma \tilde{u}-\tilde{u}), & \text { on } \Omega \backslash \Omega_{1},\end{cases} \\
\varepsilon_{n}= \begin{cases}0, \quad n=0, & \\
1, & n>0,\end{cases}
\end{gathered}
$$

$$
\begin{aligned}
& u_{h}^{(2 n+1)}= \begin{cases}u_{1 h}^{(2 n+1)}, & \text { in } \Omega_{1}, \\
u_{h}^{(2 n)}, & \text { in } \Omega \backslash \Omega_{1},\end{cases} \\
& u_{h}^{(2 n+2)}= \begin{cases}u_{h}^{(2 n+1)}, & \text { in } \Omega \backslash \Omega_{2}, \\
u_{2 h}^{(2 n+2)}, & \text { in } \Omega_{2},\end{cases}
\end{aligned}
$$

By the Poisson integral formula, the solution of (62) can be given as

$$
u_{2 h}^{(2 n+2)}=\mathscr{P} \gamma_{2} u_{h}^{(2 n+1)},
$$

where $\gamma_{2}: H^{1}\left(\Omega_{1}\right) \rightarrow H^{1 / 2}\left(\Gamma_{2}\right)$ denotes the Dirichlet trace operator and $\mathscr{P}: H^{1 / 2}\left(\Gamma_{2}\right) \rightarrow H_{0}^{1}\left(\Omega_{2}\right)$ denotes the Poisson integral operator. It is easy to verify that

$$
u_{h}^{0}=\tilde{u}
$$


TABLE $1:\left(a, R_{2}, m\right)=(1,1.75,2)$.

\begin{tabular}{|c|c|c|c|c|c|c|c|c|}
\hline$N_{1}$ & $\mathrm{~N}_{2}$ & $s$ & $n$ & 0 & 1 & 2 & 3 & 4 \\
\hline \multirow{3}{*}{4} & & & $e(n)$ & $2.5231 \times 10^{-1}$ & $7.2245 \times 10^{-2}$ & $6.1215 \times 10^{-2}$ & $4.2233 \times 10^{-2}$ & $4.0159 \times 10^{-2}$ \\
\hline & 8 & 2 & $e_{h}(n)$ & & $9.8566 \times 10^{-2}$ & $2.6452 \times 10^{-2}$ & $6.1205 \times 10^{-3}$ & $1.7354 \times 10^{-3}$ \\
\hline & & & $q_{h}(n)$ & & & 3.7270 & 3.6219 & 3.5267 \\
\hline \multirow{3}{*}{8} & & & $e(n)$ & $2.1628 \times 10^{-1}$ & $6.8132 \times 10^{-2}$ & $4.2354 \times 10^{-2}$ & $2.1532 \times 10^{-2}$ & $2.1533 \times 10^{-2}$ \\
\hline & 16 & 4 & $e_{h}(n)$ & & $1.5982 \times 10^{-1}$ & $4.5357 \times 10^{-2}$ & $1.2986 \times 10^{-2}$ & $3.7281 \times 10^{-3}$ \\
\hline & & & $q_{h}(n)$ & & & 3.5236 & 3.4926 & 3.4432 \\
\hline \multirow{3}{*}{16} & & & $e(n)$ & $1.8437 \times 10^{-1}$ & $3.5468 \times 10^{-2}$ & $2.3459 \times 10^{-2}$ & $2.0245 \times 10^{-2}$ & $1.7342 \times 10^{-2}$ \\
\hline & 32 & 8 & $e_{h}(n)$ & & $1.2183 \times 10^{-1}$ & $3.7647 \times 10^{-2}$ & $1.1743 \times 10^{-2}$ & $3.6502 \times 10^{-3}$ \\
\hline & & & $q_{h}(n)$ & & & 3.2361 & 3.2058 & 3.2172 \\
\hline
\end{tabular}

TABLE 2: $\left(a, R_{2}, m\right)=(1,1.75,2)$.

\begin{tabular}{|c|c|c|c|c|c|c|c|c|}
\hline$N_{1}$ & $N_{2}$ & $s$ & $n$ & 5 & 6 & 7 & 8 & 9 \\
\hline \multirow{3}{*}{4} & & & $e(n)$ & $4.0029 \times 10^{-2}$ & $3.9021 \times 10^{-2}$ & $3.9002 \times 10^{-2}$ & $3.8876 \times 10^{-2}$ & $3.8651 \times 10^{-2}$ \\
\hline & 8 & 2 & $e_{h}(n)$ & $5.2202 \times 10^{-4}$ & $1.7305 \times 10^{-4}$ & $5.7476 \times 10^{-5}$ & $1.9094 \times 10^{-5}$ & $6.2964 \times 10^{-6}$ \\
\hline & & & $q_{h}(n)$ & 3.3245 & 3.0166 & 3.0108 & 3.0102 & 3.0326 \\
\hline \multirow{3}{*}{8} & & & $e(n)$ & $2.0522 \times 10^{-2}$ & $2.0614 \times 10^{-2}$ & $1.8631 \times 10^{-2}$ & $1.7532 \times 10^{-2}$ & $1.7092 \times 10^{-2}$ \\
\hline & 16 & 4 & $e_{h}(n)$ & $1.1646 \times 10^{-3}$ & $3.7540 \times 10^{-4}$ & $1.2175 \times 10^{-4}$ & $3.9789 \times 10^{-5}$ & $1.3206 \times 10^{-5}$ \\
\hline & & & $q_{h}(n)$ & 3.2012 & 3.1823 & 3.0832 & 3.0601 & 3.0129 \\
\hline \multirow{3}{*}{16} & & & $e(n)$ & $6.9625 \times 10^{-3}$ & $3.8703 \times 10^{-3}$ & $2.7213 \times 10^{-3}$ & $2.8173 \times 10^{-3}$ & $2.6785 \times 10^{-3}$ \\
\hline & 32 & 8 & $e_{h}(n)$ & $1.1663 \times 10^{-3}$ & $3.7108 \times 10^{-4}$ & $1.2291 \times 10^{-4}$ & $4.0792 \times 10^{-5}$ & $1.3538 \times 10^{-5}$ \\
\hline & & & $q_{h}(n)$ & 3.1295 & 3.1432 & 3.0190 & 3.0132 & 3.0130 \\
\hline
\end{tabular}

Theorem 7. For the discrete Schwartz alternating algorithms (61)-(62) and the constant $\alpha$ in Theorem 4, there hold the following error estimates

$$
\begin{aligned}
& \left|u-u_{h}^{(2 n+1)}\right|_{1} \leqslant C h+\alpha^{2 n}\left|u_{h}^{*}-u_{h}^{(1)}\right|_{1}, \\
& \left|u-u_{h}^{(2 n+2)}\right|_{1} \leqslant C h+\alpha^{2 n+2}\left|u_{h}^{*}-u_{h}^{(0)}\right|_{1} .
\end{aligned}
$$

Since $u-u_{h}^{(n)} \in V$, (70) also hold true if the norm in their left hand sides is replaced by the norm of $H_{0}^{1}(\Omega)$.

\section{Numerical Examples}

To test the effectiveness of the method in this paper, we give some numerical examples, using the discrete Schwartz alternating algorithm in Section 5. In all examples, the exact solutions are known. The purpose of these examples is to check the convergence in terms of iteration and mesh size $h$.

Suppose that $\Omega^{c}=\{(r, \theta, \varphi)|| r \mid>a, 0 \leqslant \theta \leqslant \pi, 0 \leqslant$ $\varphi \leqslant 2 \pi\}$ is the exterior domain of sphere $x^{2}+y^{2}+z^{2}=a^{2}$. The artificial boundary is $\Gamma_{1}=\{(r, \theta, \varphi)|| r \mid=m a, 0 \leqslant \theta \leqslant$ $\pi, 0 \leqslant \varphi \leqslant 2 \pi\}\left(m>1, m \in Z^{+}\right), \Gamma_{2}=\{(r, \theta, \varphi)|| r \mid=$ $\left.R_{2}, 0 \leqslant \theta \leqslant \pi, 0 \leqslant \varphi \leqslant 2 \pi\right\}$. Split the bounded domain $\Omega_{1}$ into finite element mesh as follows. We subdivide the bounded domain $\Omega_{1}$ using the following family of planes $\theta=$ $\left(\pi / N_{1}\right) j\left(j=0,1, \ldots, N_{1}\right), \varphi_{\ell}=\left(2 \pi / N_{2}\right) \ell\left(\ell=0,1, \ldots, N_{2}\right)$, and $r=i a / s(i=s, s+1, \ldots, m s)$, where $s$ is a positive integer. Now we obtain a uniform hexahedrons partition of $\Omega_{1}$ with mesh size of $h=a / s$. Let $S_{h}\left(\Omega_{1}\right)$ be the piecewise trilinear finite element space on $\Omega_{1}$. Substitute

$$
\tilde{u}^{I}=\sum_{P_{i} \in \sum_{0}} g_{0}\left(P_{i}\right) L_{i}(\mathbf{x})
$$

for $\tilde{u}$ (where $L_{i}(\mathbf{x})$ denotes the interpolation basis function corresponding to node $P_{i}$ ).

Denote $e$ the maximum node-error on $\bar{\Omega}_{i}$

$$
e(n)=\sup _{P_{i} \in \bar{\Omega}_{i}}\left|u\left(P_{i}\right)-u_{1 h}^{(2 n)}\left(P_{i}\right)\right| ;
$$

$e_{h}$ denotes the maximum node-error of the adjacent twosteps on nodes

$$
e_{h}(n)=\sup _{P_{i} \in \bar{\Omega}_{i}}\left|u_{1 h}^{(2 n+2)}\left(P_{i}\right)-u_{1 h}^{(2 n)}\left(P_{i}\right)\right|
$$

$q_{h}$ is the convergence rate $q_{h}(n)=e_{h}(n-1) / e_{h}(n)$. We substitute $\sum_{\ell=0}^{M}$ for $\sum_{\ell=0}^{\infty}$ in the computing of the entries of stiffness matrix.

Example 1. We take that the exact solution of problem (1) is

$$
u(r, \theta, \varphi)=-\sqrt{\frac{3}{4 \pi}} \frac{k r+\mathrm{i}}{k^{2} r^{2}} e^{\mathrm{i} k r} \cos \theta
$$

where

$$
g=-\sqrt{\frac{3}{4 \pi}} \frac{k+\mathrm{i}}{k^{2}} e^{\mathrm{i} k} \cos \theta
$$

Let $k=2.0, a=1, m=2, R_{2}=1.75$, and $M=4$; the numerical results are shown in Tables 1 and 2 .

Example 2. We take that the exact solution of problem (1) is

$$
\begin{aligned}
& u(r, \theta, \varphi) \\
& \quad=-\sqrt{\frac{15}{32 \pi}}\left(\frac{3 \mathrm{i}}{k^{3} r^{3}}+\frac{3}{k^{2} r^{2}}-\frac{\mathrm{i}}{k r}\right) e^{\mathrm{i}(k r+\varphi)} \sin 2 \theta,
\end{aligned}
$$


TABLE 3: $\left(a, R_{2}, m\right)=(1,1.85,2)$.

\begin{tabular}{|c|c|c|c|c|c|c|c|c|}
\hline$N_{1}$ & $\mathrm{~N}_{2}$ & $s$ & $n$ & 0 & 1 & 2 & 3 & 4 \\
\hline \multirow{3}{*}{4} & & & $e(n)$ & $2.5231 \times 10^{-1}$ & $5.2541 \times 10^{-2}$ & $5.0562 \times 10^{-2}$ & $4.5623 \times 10^{-2}$ & $3.8128 \times 10^{-2}$ \\
\hline & 8 & 2 & $e_{h}(n)$ & & $7.5434 \times 10^{-1}$ & $2.1411 \times 10^{-2}$ & $6.6464 \times 10^{-3}$ & $2.1131 \times 10^{-3}$ \\
\hline & & & $q_{h}(n)$ & & & 3.5231 & 3.2215 & 3.1452 \\
\hline \multirow{3}{*}{8} & & & $e(n)$ & $2.1628 \times 10^{-1}$ & $6.2315 \times 10^{-2}$ & $5.1532 \times 10^{-2}$ & $3.8321 \times 10^{-2}$ & $2.2532 \times 10^{-2}$ \\
\hline & 16 & 4 & $e_{h}(n)$ & & $1.4526 \times 10^{-1}$ & $4.4753 \times 10^{-2}$ & $1.4856 \times 10^{-2}$ & $5.0306 \times 10^{-3}$ \\
\hline & & & $q_{h}(n)$ & & & 3.2458 & 3.0124 & 2.9532 \\
\hline \multirow{3}{*}{16} & & & $e(n)$ & $1.8437 \times 10^{-1}$ & $2.4623 \times 10^{-2}$ & $2.3245 \times 10^{-2}$ & $2.1244 \times 10^{-2}$ & $2.0132 \times 10^{-2}$ \\
\hline & 32 & 8 & $e_{h}(n)$ & & $1.0157 \times 10^{-1}$ & $3.6381 \times 10^{-2}$ & $1.4196 \times 10^{-2}$ & $5.7773 \times 10^{-3}$ \\
\hline & & & $q_{h}(n)$ & & & 2.7918 & 2.5628 & 2.4572 \\
\hline
\end{tabular}

TABle $4:\left(a, R_{2}, m\right)=(1,1.85,2)$.

\begin{tabular}{|c|c|c|c|c|c|c|c|c|}
\hline$N_{1}$ & $\mathrm{~N}_{2}$ & $s$ & $n$ & 5 & 6 & 7 & 8 & 9 \\
\hline \multirow{3}{*}{4} & \multirow{3}{*}{8} & \multirow{3}{*}{2} & $e(n)$ & $3.7170 \times 10^{-2}$ & $3.5319 \times 10^{-2}$ & $3.4321 \times 10^{-2}$ & $3.2112 \times 10^{-2}$ & $2.9518 \times 10^{-2}$ \\
\hline & & & $e_{h}(n)$ & $2.3888 \times 10^{-4}$ & $9.6127 \times 10^{-5}$ & $4.1760 \times 10^{-5}$ & $1.8519 \times 10^{-5}$ & $8.2215 \times 10^{-6}$ \\
\hline & & & $q_{h}(n)$ & 2.5621 & 2.4851 & 2.3019 & 2.2549 & 2.2526 \\
\hline \multirow{3}{*}{8} & \multirow{3}{*}{16} & \multirow{3}{*}{4} & $e(n)$ & $2.0213 \times 10^{-2}$ & $1.9526 \times 10^{-2}$ & $1.9432 \times 10^{-2}$ & $1.9542 \times 10^{-2}$ & $1.8926 \times 10^{-2}$ \\
\hline & & & $e_{h}(n)$ & $1.3314 \times 10^{-3}$ & $4.8891 \times 10^{-4}$ & $2.1574 \times 10^{-4}$ & $9.5621 \times 10^{-5}$ & $4.2462 \times 10^{-5}$ \\
\hline & & & $q_{h}(n)$ & 2.8001 & 2.7232 & 2.2662 & 2.2572 & 2.2519 \\
\hline \multirow{3}{*}{16} & \multirow{3}{*}{32} & \multirow{3}{*}{8} & $e(n)$ & $1.9524 \times 10^{-2}$ & $9.5324 \times 10^{-3}$ & $8.4526 \times 10^{-3}$ & $8.0219 \times 10^{-3}$ & $7.8243 \times 10^{-3}$ \\
\hline & & & $e_{h}(n)$ & $1.4861 \times 10^{-3}$ & $6.4006 \times 10^{-4}$ & $2.8198 \times 10^{-4}$ & $1.2506 \times 10^{-4}$ & $5.5502 \times 10^{-5}$ \\
\hline & & & $q_{h}(n)$ & 2.4562 & 2.3218 & 2.2699 & 2.2548 & 2.2532 \\
\hline
\end{tabular}

where

$$
g=-\sqrt{\frac{15}{32 \pi}} \frac{3 \mathrm{i}+3 k-\mathrm{i} k^{2}}{k^{3}} e^{\mathrm{i}(k+\varphi)} \sin 2 \theta
$$

Let $k=2.0, a=1, m=2, R_{2}=1.85$, and $M=4$; the numerical results are shown in Tables 3 and 4 .

As can be seen from Tables 3 and 4, the discrete Schwartz alternating algorithm is geometrically convergent and $q_{h}(n)$ is nearly not affected by mesh parameter $h$. With the increase of $R_{2}, q_{h}(n)$ is smaller. So the larger the overlapping part is, the faster the convergence rate will be. All these are in accord with the theoretical analyses.

\section{Conflicts of Interest}

The authors declare that they have no conflicts of interest.

\section{Acknowledgments}

The research is partly supported by the National Natural Sciences Foundation of China (Contact/Grant nos. 11401296 and 11371198), Jiangsu Provincial Natural Science Foundation of China (Contact/Grant no. BK20141008), and the Natural Science Fund for Colleges and Universities in Jiangsu Province (Contact/Grant no. 14KJB110007). And it is also partly sponsored by Qing Lan Project.

\section{References}

[1] D. Givoli, Numerical Methods for Problems in Infinite Domains, Studies in Applied Mechanics, Elsevier, Amsterdam, Netherlands, 1992.

[2] I. Harari and T. J. Hughes, "Analysis of continuous formulations underlying the computation of time-harmonic acoustics in exterior domains," Computer Methods Applied Mechanics and Engineering, vol. 97, no. 1, pp. 103-124, 1992.

[3] D. Givoli and J. B. Keller, "A finite element method for large domains," Computer Methods Applied Mechanics and Engineering, vol. 76, no. 1, pp. 41-66, 1989.

[4] R. E. Kleinman and G. F. Roach, "Boundary integral equations for the three-dimensional Helmholtz equation," SIAM Review, vol. 16, pp. 214-236, 1974.

[5] D. H. Yu, "A domain decomposition method based on the natural boundary reduction over an unbounded domain," Mathematica Numerica Sinica, vol. 16, no. 4, pp. 448-459, 1994.

[6] M. Yang and Q. Du, "A Schwarz alternating algorithm for elliptic boundary value problems in an infinite domain with a concave angle," Applied Mathematics and Computation, vol. 159, no. 1, pp. 199-220, 2004.

[7] Q. Zheng and P. Zhao, "An overlapping domain decomposition method for exterior Stokes problems," Journal on Numerical Methods and Computer Applications, vol. 31, no. 4, pp. 271-278, 2010.

[8] Z. P. Jia and D. H. Yu, "An overlapping domain decomposition method based on natural boundary reduction for a 2-D exterior Helmholtz problem," Journal on Numerical Methods and Computer Applications, vol. 22, no. 4, pp. 241-253, 2001.

[9] J. M. Wu and D. H. Yu, "The natural integral equations of 3-D exterior Helmholtz problem and its numerical solution," 
Chinese Journal of Computational Physics, vol. 16, no. 5, pp. 449456, 1999.

[10] J.-m. Wu and D.-h. Yu, "The overlapping domain decomposition method for harmonic equation over exterior threedimensional domain," Journal of Computational Mathematics, vol. 18, no. 1, pp. 83-94, 2000.

[11] Q. Chen, B. Liu, and Q. Du, "A D-N alternating algorithm for solving 3D Exterior Helmholtz problems," Mathematical Problems in Engineering, Article ID 418426, Art. ID 418426, 8 pages, 2014.

[12] Q. Chen, Domain decomposition methods for exterior Helmholtz problems [D], Nanjing Normal University, 2015. 


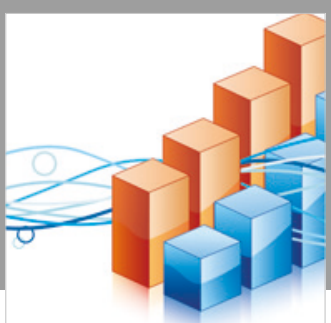

Advances in

Operations Research

\section{-n-m}
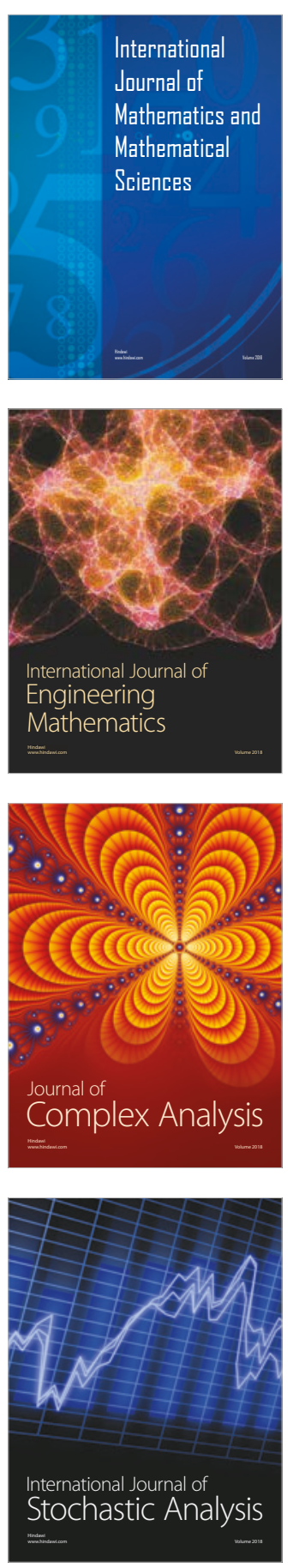
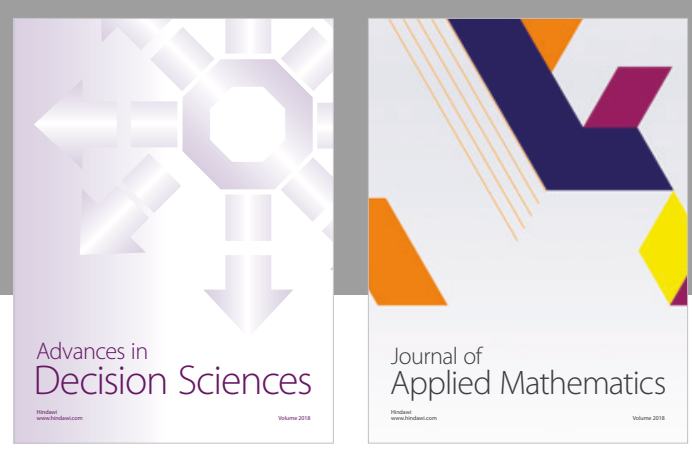

Journal of

Applied Mathematics
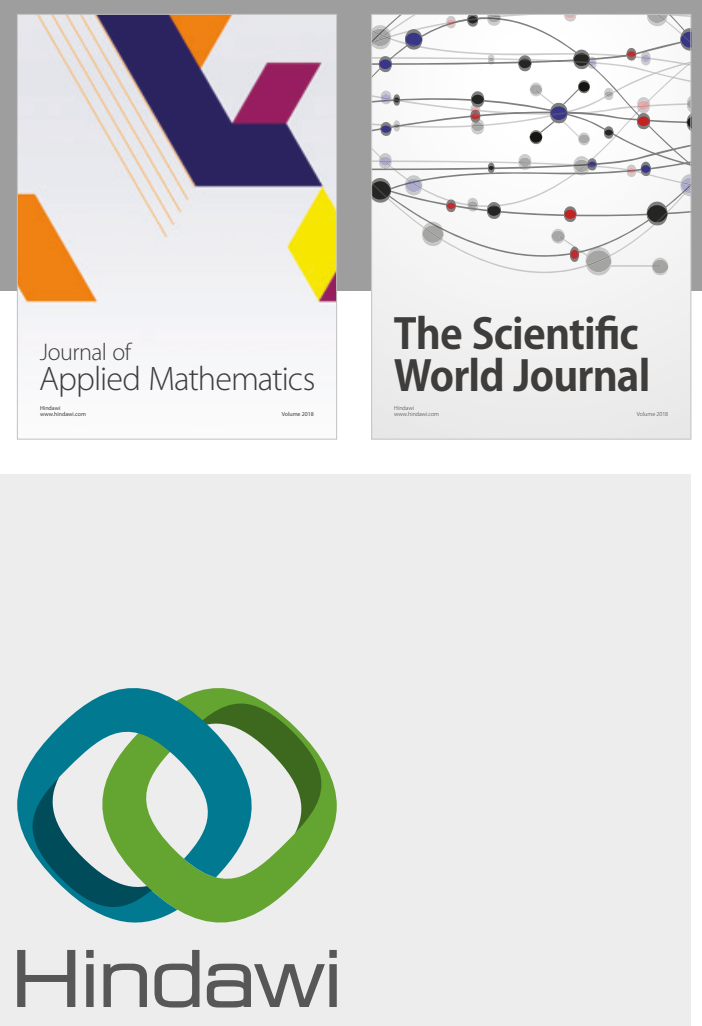

Submit your manuscripts at

www.hindawi.com

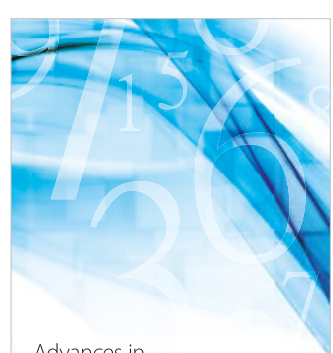

Advances in
Numerical Analysis
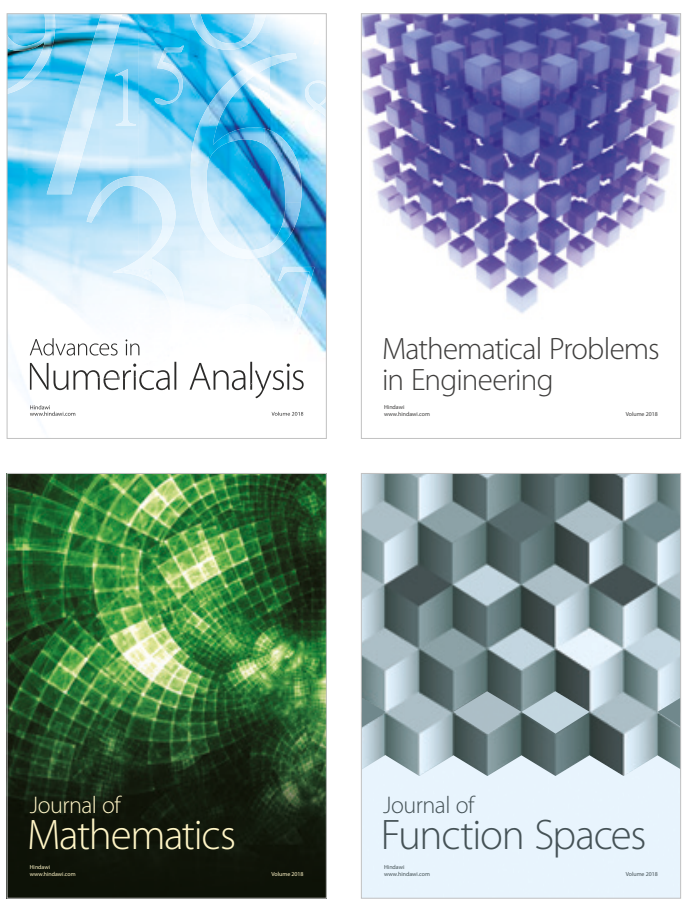

Mathematical Problems in Engineering

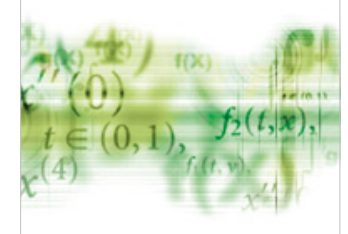

International Journal of

Differential Equations

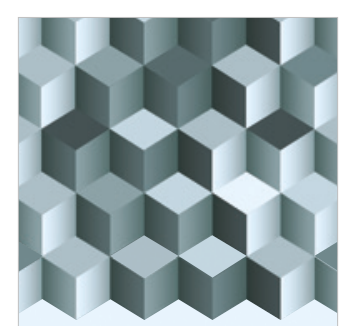

Journal of

Function Spaces

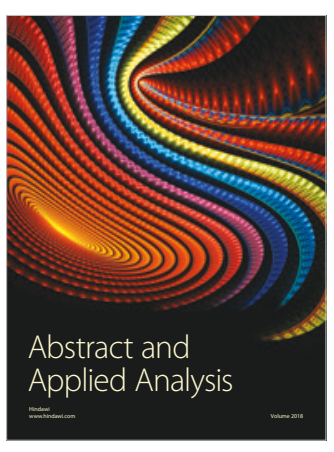

The Scientific

World Journal

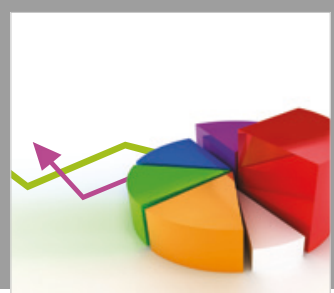

Journal of

Probability and Statistics
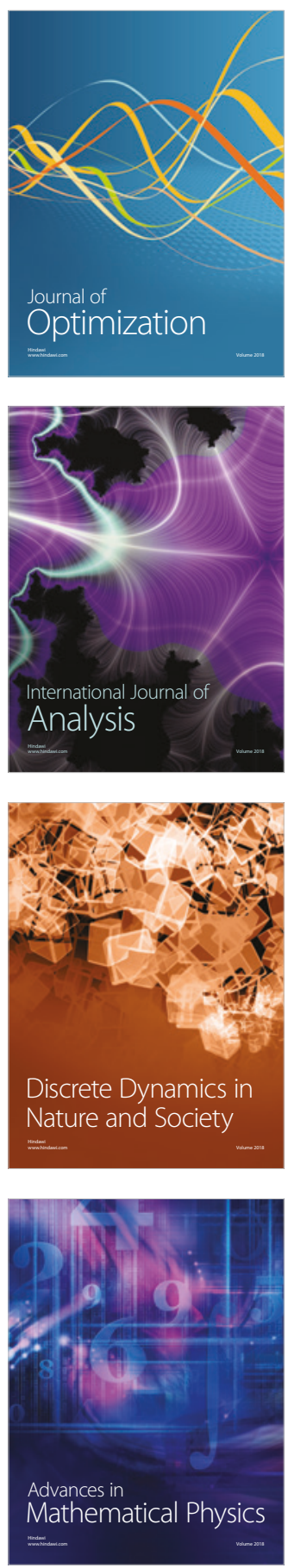